\title{
Integrated differential pressure sensor in silicon-on-insulator
}

\author{
Elewout Hallynck and Peter Bienstman \\ Photonics Research Group, Department of Information Technology (INTEC) \\ Ghent University - imec, Belgium \\ Center for Nano- and Biophotonics (NB-Photonics), Ghent University, Belgium \\ E-mail: elewout.hallynck@intec.ugent.be
}

\begin{abstract}
We have fabricated and characterized a compact integrated optical pressure sensor in silicon-on-insulator. Measurements have shown that spectral features in our device can shift up to $1585 \mathrm{pm}$ going from -20 to $80 \mathrm{kPa}$.
\end{abstract}

\section{INTRODUCTION}

Pressure sensors have many applications, e.g., determining the quality of vacuum or checking the pressure in a tire. Although plenty of research has already been done on electrical pressure sensors, it is also possible to develop these sensors in the optical domain where the key advantages are immunity to electromagnetic interference and the ability to deploy them in spark-sensitive environments. Several approaches have already been demonstrated (Fabry-Perot based [1], fiber Bragg grating based [2] or integrated sensors [3]) in a variety of material platforms. In this paper, we present a significant improvement over previously reported results by other groups [4] and ourselves [5] for an integrated pressure sensor in silicon-oninsulator.

\section{OPERATION PRINCIPLE}

The operation principle of the optical pressure sensor described here is as follows: the substrate under part of a MachZehnder interferometer on a silicon-on-insulator chip is locally thinned in order to achieve a pressure-sensitive membrane. When applying a differential pressure on the membrane, the structure will deform. This membrane deformation will induce stress which in turn causes the elasto-optic to alter the optical path length of the light in the structure, giving rise to a phase shift. This in turn will cause the position of the spectral features to shift in the wavelength spectrum. Measuring this shift gives an indication on how much the pressure has changed.

Simulations with the finite elements solver ANSYS allowed us to determine the stress caused by pressure in a $60 \mu \mathrm{m}$ by $60 \mu \mathrm{m}, 220 \mathrm{~nm}$ thin silicon membrane. As can be seen from Figure 1 we observe a non-linear response of the in plane stress $\sigma_{x x}$ in the center of the membrane to the applied pressure. We expect a similar response of the effective index shift in function of pressure.

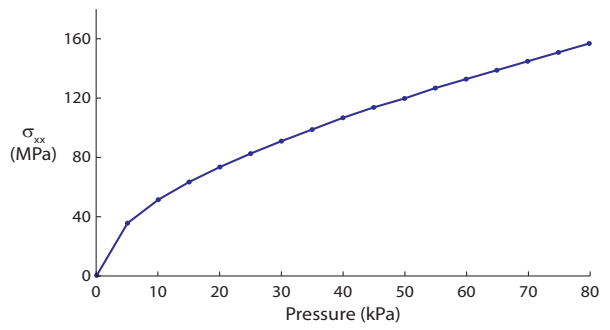

Fig. 1. The non-linear response of the in plane stress $\sigma_{x x}$ in the center of the membrane to the applied pressure.

\section{EXPERIMENTS}

\section{A. Device description}

Using a $193 \mathrm{~nm}$ deep UV lithography process, the photonic circuits are fabricated in silicon-on-insulator $(220 \mathrm{~nm}$ silicon top layer, $2 \mu \mathrm{m}$ silicon dioxide and $750 \mu \mathrm{m}$ silicon substrate). The substrate thickness is subsequently reduced from $750 \mu \mathrm{m}$ to $250 \mu \mathrm{m}$. We deposit a stack of $400 \mathrm{~nm}$ plasma enhanced chemical vapor deposited (PECVD) silicon nitride on $400 \mathrm{~nm}$ PECVD silicon dioxide on both sides of the wafer to act as either a protective layer (top side) or etching mask (bottom side). Since the silicon dioxide and silicon nitride may still contain pinholes that allow the $\mathrm{KOH}$ to penetrate to the top silicon layer, we apply an extra protective coating on the top side of the wafer using the commercially available ProTEK B3. After curing of this layer, we put the wafer in $20 \% \mathrm{KOH}$ for approximately 3 hours at a temperature of $79^{\circ} \mathrm{C}$. The $\mathrm{KOH}$ will then etch the silicon anisotropically until it practically stops at the silicon dioxide layer. After silicon etching, the ProTEK top layer is removed using its dedicated remover while residues and the ProTEK primer layer are removed using both oxygen plasma cleaning and wet piranha etching $\left(3 \mathrm{H}_{2} \mathrm{SO}_{4}: 1 \mathrm{H}_{2} \mathrm{O}_{2}\right)$. A 20 -s dip in $49 \% \mathrm{HF}$ further thins down the membrane by etching away the top silicon nitride and silicon dioxide layers. SEM measurements show that the final membranes consist of $220 \mathrm{~nm}$ silicon on $1.8 \mu \mathrm{m}$ buried silicon dioxide, bringing the total membrane thickness to $2.02 \mu \mathrm{m}$.

\section{B. Setup description}

To apply pressure to the membrane, a chuck was designed on which the sensor is mechanically clamped while the pres- 
sure difference can be applied through a hole in the chuck that needs to be positioned under the membrane. The pressure difference is then obtained by means of a piston that can change the volume of the pressure circuit. To ensure that we maintain a fixed pressure, we include a feedback mechanism: a digital pressure sensor triggers a pump when the pressure drops below a threshold.

In the wavelenght spectrum, an intensity peak in the vicinity of $1.55 \mu \mathrm{m}$ is selected to be monitored. A $3-\mathrm{nm}$ window around this peak is then swept using a tunable laser with a step size of $5 \mathrm{pm}$. Pressure is applied and the shifted position of the peak is determined. The pressure is increased in steps of generally $10 \mathrm{kPa}$ and each time a new measurement is performed. Data analysis consists of fitting a squared cosine [3] to the measured spectra.

\section{Measurements}

Figure 2 shows the device that has been investigated. In Figure 3 the shift of one of the spectral features under influence of pressure is displayed. Opposed to [5] we were also able to measure pressure that caused the membrane to be pushed outward (i.e. pressure under membrane is greater than outside pressure; negative side of $\mathrm{x}$-axis). If we compare the shape of the response curve of Figure 3 to that of Figure 1, we notice that the initial steep part of Figure 1 is not visible in Figure 3. This is most likely caused by the fact that there is already some initial stress present in the sensor which was not taken into account in the simulations. Since the curve is not linear, it is not possible to extract a sensitivity per unit of pressure but over a range of $100 \mathrm{kPa}(-20 \mathrm{kPa}$ to $80 \mathrm{kPa})$ the monitored intensity peak shifts by $755 \mathrm{pm}$. If we then shortly perform another HF dip (10 s) to further reduce the thickness of the membrane we see, as expected, an increased sensitivity: the peak now shifts by $1585 \mathrm{pm}$.

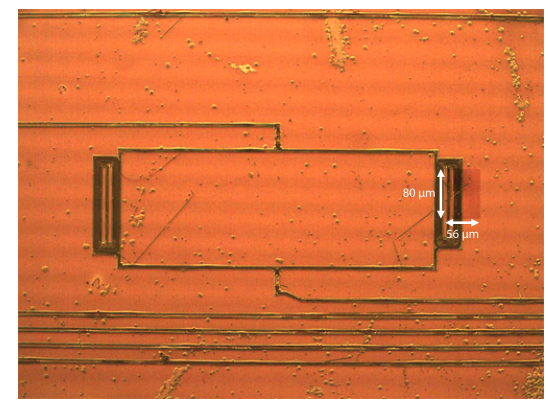

Fig. 2. Microscope of the integrated Mach-Zehnder inteferometer used for pressure sensing. The membrane is located in the discolored region.

\section{Discussion}

Although the described sensor already shows a high sensitivity, considering its small footprint, there is still room for improvement. It has been shown that a membrane where the length/width ratio equals 2 will exhibit higher sensitivity [6]. Secondly, in [6] it is shown that using TM polarized light will enhance the sensitivity since the refractive index change in

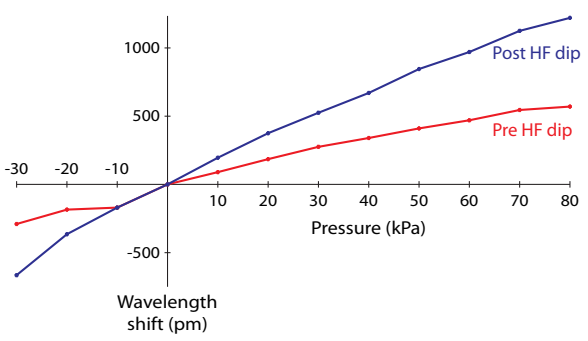

Fig. 3. Position of a spectral dip in function of the pressure applied to a Mach-Zehnder interferometer on a membrane. The total shift from -20 to 80 $\mathrm{kPa}$ is $755 \mathrm{pm}$ (Pre HF dip). This can be further increased to $1585 \mathrm{pm}$ (Post HF dip).

the out of plane direction is greater than it is in plane. Lastly, placing several membranes in series (as demonstrated by [3]) will also increase overall sensitivity of the device.

\section{CONClusions}

We have demonstrated a pressure sensor in silicon-oninsulator with a relatively thin pressure-sensitive membrane. The high refractive index contrast in silicon-on-insulator, combined with the small membrane thickness, permits us to fabricate highly sensitive sensors with a small footprint (80 $\mu \mathrm{m} \times 56 \mu \mathrm{m})$. Due to the non-linear response of the sensor, it is not straightforward to determine a sensitivity per units of pressure but a shift of spectral features of $755 \mathrm{pm}$ and $1585 \mathrm{pm}$ (depending on the thickness of the membrane) for a differential pressure range of $100 \mathrm{kPa}$ has been demonstrated, which is in the same order of magnitude as in [3] where much larger membranes are used (order of millimeters).

\section{ACKNOWLEDGEMENT}

The authors would like to thank S. Verstuyft for the material deposition, P. Guns for fabricating the mechanical holder, T. Claes for designing the optical structures and L. Van Landschoot for the SEM measurements. This work has been funded through an imec research grant.

\section{REFERENCES}

[1] F. Xu, D. Ren, X. Shi, C. Li, W. Lu, L. Lu, L. Lu, and B. Yu, "High-sensitivity Fabry-Perot interferometric pressure sensor based on a nanothick silver diaphragm," Optics Letters, vol. 37, no. 2, pp. 133-135, 2012.

[2] D. A. Singlehurst, C. R. Dennison, and P. M. Wild, "Comprising Multiplexed In-Fibre Bragg Gratings Within a Flexible Superstructure," Journal of Lightwave Technology, vol. 30, no. 1, pp. 123-129, 2012.

[3] H. Porte, V. Gorel, S. Kiryenko, J.-P. Goedgebuer, W. Daniau, and P. Blind, "Imbalanced Mach-Zehnder interferometer integrated in micromachined silicon substrate for pressure sensor," Journal of Lightwave Technology, vol. 17, no. 2, pp. 229-233, 1999.

[4] X. Zhao, J. M. Tsai, H. Cai, X. M. Ji, J. Zhou, M. H. Bao, Y. P. Huang, D. L. Kwong, and a. Q. Liu, "A nano-opto-mechanical pressure sensor via ring resonator," Optics Express, vol. 20, no. 8, p. 8535, Mar. 2012.

[5] E. Hallynck and P. Bienstman, "Integrated Optical Pressure Sensors in Silicon-on-Insulator," IEEE Photonics Journal, vol. 4, no. 2, pp. 443-450, 2012.

[6] M. Ohkawa, M. Izutsu, and T. Sueta, "Integrated optic pressure sensor on silicon substrate." Applied Optics, vol. 28, no. 23, pp. 5153-5157, Dec. 1989. 